\title{
A bound for orderings of Reidemeister moves
}

\author{
JULIAN GOLD
}

\begin{abstract}
We provide an upper bound on the number of ordered Reidemeister moves required to pass between two diagrams of the same link. This bound is in terms of the number of unordered Reidemeister moves required.
\end{abstract}

$57 \mathrm{M} 25$

In 1927 Kurt Reidemeister proved that any two link diagrams representing the same link may be joined by a finite sequence of Reidemeister moves. The importance of this theorem to knot theory cannot be overstated. Mathematicians like Alexander Coward [1; 2], Marc Lackenby [2], Bruce Trace [4], and Joel Hass and Jeffery Lagarias [3] have all explored properties of sequences of Reidemeister moves.
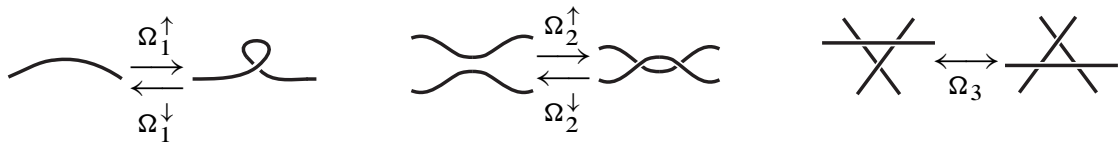

Figure 1: Reidemeister moves

In 2006, Alexander Coward showed in [1] that given any sequence of Reidemeister moves between link diagrams $D_{1}$ and $D_{2}$, it is possible to construct a new sequence ordered in the following way: first $\Omega_{1}^{\uparrow}$ moves, then $\Omega_{2}^{\uparrow}$ moves, then $\Omega_{3}$ moves, finally $\Omega_{2}^{\downarrow}$ moves. We present, via the following theorem, an upper bound on the number of moves required for an ordered sequence in terms of the number of moves present in any sequence of Reidemeister moves.

Theorem 1 Let $D_{1}$ and $D_{2}$ be diagrams for the same link that are joined by a sequence of $M$ Reidemeister moves. Let $N=6^{M+1} M$. Then there exists a sequence of no more than $\exp ^{(N)}(N)$ moves from $D_{1}$ to $D_{2}$ ordered in the following way: first $\Omega_{1}^{\uparrow}$, then $\Omega_{2}^{\uparrow}$, then $\Omega_{3}$, then $\Omega_{2}^{\downarrow}$ and finally $\Omega_{1}^{\downarrow}$.

Here the function exp is defined as $\exp (x)=2^{x}$ and $\exp ^{(r)}(x)$ is the function exp iterated $r$ times on input $x$.

We define a link diagram to be a 4-valent graph embedded in $\mathbb{R}^{2}$ with crossing information recorded at each vertex. All diagrams will be oriented, so that they 
represent oriented links. We regard two diagrams as the same if there is an ambient isotopy of $\mathbb{R}^{2}$ taking one diagram to the other, preserving crossing information and the orientation of each link component. To prove Theorem 1, we will adapt the methods Alexander Coward uses in [1] and borrow the following terminology.

Definition Let $D$ be a link diagram and suppose $c:[0,1] \rightarrow \mathbb{R}^{2}$ is an embedded path whose image $C$ intersects $D$ transversely at finitely many points, where $c(0) \in D$ and $c(1) \notin D$. We stipulate that no point of intersection of $D$ and $C$ is a vertex of $D$. At each such point, apart from $c(0)$, we designate whether $C$ passes over or under $D$.

Let $C \times[-\epsilon, \epsilon]$ be a small neighborhood of $C$ such that

$$
(C \times[-\epsilon, \epsilon]) \cap D=(C \cap D) \times[-\epsilon, \epsilon] .
$$

Then define the diagram $D^{\prime}$ as the 4-valent graph

$$
D \cup \partial(C \times[-\epsilon, \epsilon]) \backslash(c(0) \times(-\epsilon, \epsilon))
$$

with crossing information induced by the path $c$. We write $D \rightsquigarrow D^{\prime}$ and say that $D^{\prime}$ is obtained from $D$ by adding a tail along $C$. Additionally, we will call $C$ the core of this tail. We require that adding a tail to a diagram $D$ produces a diagram $D^{\prime}$ where $c\left(D^{\prime}\right)>c(D)$. Figure 2 illustrates the construction of a tail.
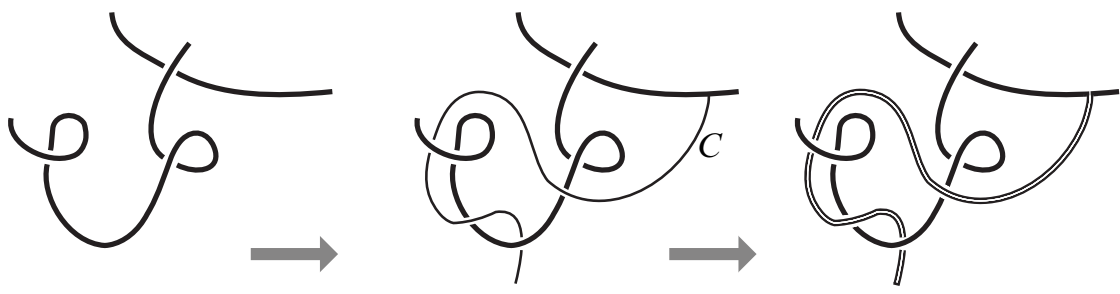

Figure 2: Adding a tail

Definition Suppose $D_{1} \rightsquigarrow D_{2}$ via some path $c:[0,1] \rightarrow \mathbb{R}^{2}$. Suppose additionally that $c(1)$ lies in a small neighborhood of some crossing $\chi$ of $D_{1}$. Let $D_{3}$ be as in Figure 3, a diagram obtained from $D_{2}$ by performing two $\Omega_{2}^{\uparrow}$ moves followed by one $\Omega_{3}$ move.

We say $D_{3}$ is obtained from $D_{1}$ by adding a lollipop and write $D_{1} \bigcirc \rightarrow D_{3}$. The lollipop itself is defined as $\overline{D_{3} \backslash D_{1}}$. The tail part of the lollipop is $\overline{\left(D_{3} \cap D_{2}\right) \backslash D_{1}}$, and the closure of the rest of the lollipop is the circle part of the lollipop. We say that the lollipop is centered at $\chi$. 


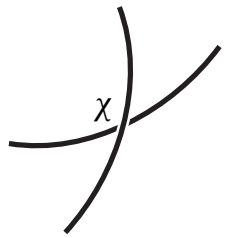

$D_{1}$

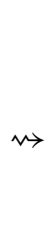

Figure 3: Adding a lollipop

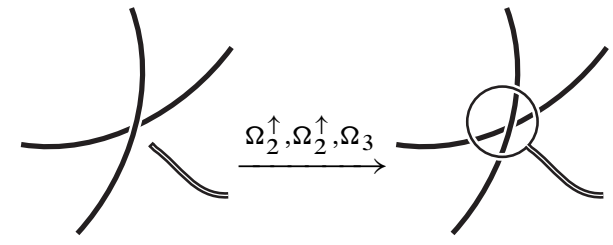

$D_{3}$

We think of a sequence $\mathcal{S}$ of Reidemeister moves, tails and lollipops between link diagrams $L_{1}$ and $L_{2}$ in the following way:

$$
\mathcal{S}: L_{1}=D_{0} \stackrel{a_{1}}{\longrightarrow} D_{1} \stackrel{a_{2}}{\longrightarrow} \cdots \stackrel{a_{n}}{\longrightarrow} D_{n}=L_{2}
$$

Here each $a_{i}$ is a Reidemeister move, a tail or a lollipop. A tail or lollipop may be added from $D_{i}$ to $D_{i+1}\left(\right.$ eg $D_{i} \rightsquigarrow D_{i+1}$ ) or from $D_{i+1}$ to $D_{i}$ (eg $D_{i} \rightsquigarrow D_{i+1}$ ). We say the length of $\mathcal{S}$ is $n$. The intermediate link diagrams $D_{i}$ are often omitted from the figures in this paper for clarity, but are implicit in any sequence.

If a link diagram $D_{2}$ is reached from $D_{1}$ by a sequence of $\Omega_{2}^{\uparrow}$ moves of length $n$, we write $D_{1} \rightarrow{ }^{n} D_{2}$. The following lemma allows us to take a sequence $\mathcal{S}$ and produce a sequence $\mathcal{S}^{\prime}$ with one less $\Omega_{3}$ move.

Lemma 2 Let $D_{1}$ and $D_{2}$ be link diagrams such that $D_{1} \stackrel{\Omega_{3}}{\longrightarrow} D_{2}$. Then there exists a diagram $D_{3}$ such that $D_{1} \rightarrow^{2} D_{3}$ and $D_{2} \bigcirc \rightarrow D_{3}$.

Proof The diagram

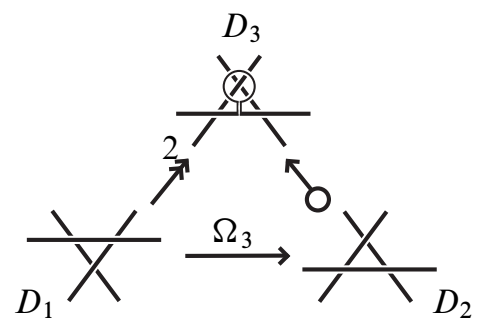

satisfies the required conditions.

If an $\Omega_{3}$ move occurs in a sequence of Reidemeister moves, tails and lollipops

$$
\mathcal{S}: A \rightarrow \cdots \rightarrow B \stackrel{\Omega_{3}}{\longrightarrow} C \rightarrow \cdots \rightarrow D
$$


we may apply Lemma 2 to $\mathcal{S}$ to get a new sequence

$$
\mathcal{S}^{\prime}: A \rightarrow \cdots \rightarrow B \stackrel{\Omega_{2}^{\uparrow}}{\longrightarrow} B^{\prime} \stackrel{\Omega_{2}^{\uparrow}}{\longrightarrow} B^{\prime \prime} \leftarrow C \rightarrow \cdots \rightarrow D .
$$

When we apply Lemma 2 to construct $\mathcal{S}^{\prime}$ from $\mathcal{S}$, we call this capping the $\Omega_{3}$ move from $B$ to $C$. The following proposition and its corollary will also allow us to build new sequences from old ones in a useful way.

Proposition 3 Suppose $D_{1} \rightsquigarrow D_{1}^{\prime}$ (or $D_{1} \bigcirc \rightarrow D_{1}^{\prime}$ ) and also that $D_{1} \rightarrow^{1} D_{2}$. Then there exists a diagram $D_{2}^{\prime}$ such that $D_{2} \rightsquigarrow D_{2}^{\prime}\left(D_{2} \bigcirc \rightarrow D_{2}^{\prime}\right.$ respectively) and $D_{1}^{\prime} \rightarrow^{a}$ $D_{2}^{\prime}$, where

$$
\begin{gathered}
c\left(D_{2}^{\prime}\right)-c\left(D_{2}\right) \leq 2\left(c\left(D_{1}^{\prime}\right)-c\left(D_{1}\right)\right) \\
a \leq c\left(D_{1}^{\prime}\right)-c\left(D_{1}\right) .
\end{gathered}
$$
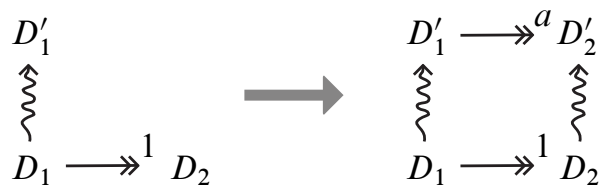

Proof The diagram $D_{2}$ is obtained from $D_{1}$ by a single $\Omega_{2}^{\uparrow}$ move which takes place over two (possibly non-distinct) edges $e_{1}$ and $e_{2}$ of $D_{1}$. Pick points $p_{1}$ and $p_{2}$ on $e_{1}$ and $e_{2}$ respectively, so that $p_{1}$ and $p_{2}$ lie outside a small neighborhood of the tail $D_{1} \rightsquigarrow D_{1}^{\prime}$. We can perform the $\Omega_{2}^{\uparrow}$ move from $D_{1}$ to $D_{2}$ by adding a tail along a path $\gamma$, which starts at $p_{1}$ and ends slightly beyond $p_{2}$.

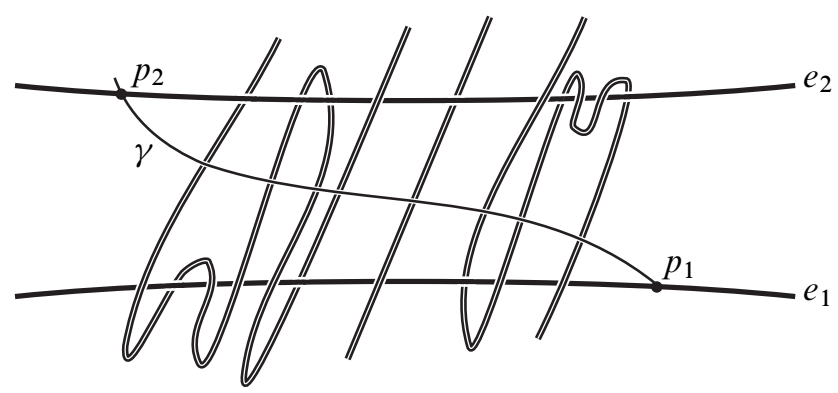

Figure 4: Constructing $D_{2}^{\prime}$ by adding a tail along $\gamma$

Diagram $D_{1}^{\prime}$ contains the points $p_{1}$ and $p_{2}$. We may arrange that the intersection of $\gamma$ with the tail $D_{1} \rightsquigarrow D_{1}^{\prime}$ contains at most $2\left\lfloor\left(c\left(D_{1}^{\prime}\right)-c\left(D_{1}\right)\right) / 4\right\rfloor$ points. Figure 4 
depicts such an arrangement. Adding a tail along $\gamma$, we construct a diagram $D_{2}^{\prime}$ with

$$
c\left(D_{2}^{\prime}\right)-c\left(D_{1}^{\prime}\right) \leq 4\left\lfloor\frac{c\left(D_{1}^{\prime}\right)-c\left(D_{1}\right)}{4}\right\rfloor+2 .
$$

Hence

$$
c\left(D_{2}^{\prime}\right)-c\left(D_{1}^{\prime}\right) \leq c\left(D_{1}^{\prime}\right)-c\left(D_{1}\right)+2 .
$$

We note that $c\left(D_{1}^{\prime}\right)-c\left(D_{1}\right)+2 \leq 2\left(c\left(D_{1}^{\prime}\right)-c\left(D_{1}\right)\right)$, because adding a tail to a diagram must raise its crossing number by at least two. This implies the desired bound on $a$. Also

$$
c\left(D_{2}^{\prime}\right)-c\left(D_{1}^{\prime}\right) \leq c\left(D_{1}^{\prime}\right)-c\left(D_{1}\right)+2
$$

implies, by adding $c\left(D_{1}^{\prime}\right)$ to both sides and subtracting $c\left(D_{2}\right)$, that

$$
c\left(D_{2}^{\prime}\right)-c\left(D_{2}\right) \leq 2 c\left(D_{1}^{\prime}\right)-c\left(D_{1}\right)+2-c\left(D_{2}\right) .
$$

Using $c\left(D_{2}\right)=c\left(D_{1}\right)+2$ we get

$$
c\left(D_{2}^{\prime}\right)-c\left(D_{2}\right) \leq 2 c\left(D_{1}^{\prime}\right)-2 c\left(D_{1}\right) .
$$

In the case that $D_{1} \bigcirc \rightarrow D_{1}^{\prime}$, choose $p_{1}$ and $p_{2}$ to be outside the circle part of the lollipop, and the above considerations go through.

Corollary 4 is a natural generalization of Proposition 3.

Corollary 4 Suppose $D_{1} \rightsquigarrow D_{1}^{\prime}$ (or $D_{1} \bigcirc \rightarrow D_{1}^{\prime}$ ) and also that $D_{1} \rightarrow^{n} D_{2}$. Then there exists a diagram $D_{2}^{\prime}$ such that $D_{2} \rightsquigarrow D_{2}^{\prime}\left(D_{1} \bigcirc \rightarrow D_{1}^{\prime}\right.$ respectively) and $D_{1}^{\prime} \rightarrow{ }^{b} D_{2}^{\prime}$, where

$$
b \leq 2^{n}\left(c\left(D_{1}^{\prime}\right)-c\left(D_{1}\right)\right) \text {. }
$$

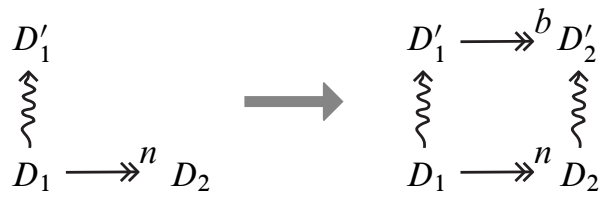

Proof Let $D_{1}, D_{2}$ and $D_{1}^{\prime}$ be as in the statement of the theorem. We work in the case $D_{1} \rightsquigarrow D_{1}^{\prime}$, but the proof for lollipops is identical. Let $\mathcal{E}$ be the sequence of $\Omega_{2}^{\uparrow}$ moves of length $n$ from $D_{1}$ to $D_{2}$,

$$
\mathcal{E}: D_{1}=E_{0} \rightarrow^{1} E_{1} \rightarrow^{1} \cdots \rightarrow^{1} E_{n}=D_{2},
$$

and let $E_{0}^{\prime}=D_{1}^{\prime}$. We use Proposition 3 to construct a diagram $E_{1}^{\prime}$ such that $E_{1} \rightsquigarrow E_{1}^{\prime}$ and $E_{0}^{\prime} \rightarrow^{b_{0}} E_{1}^{\prime}$, where $b_{0} \leq c\left(E_{0}^{\prime}\right)-c\left(E_{0}\right)$. Apply Proposition 3 again to the triple $\left(E_{1}, E_{1}^{\prime}, E_{2}\right)$ to build a diagram $E_{2}^{\prime}$. Iterate this, constructing the diagrams $E_{2}^{\prime}$ through $E_{n}^{\prime}$, as below. 


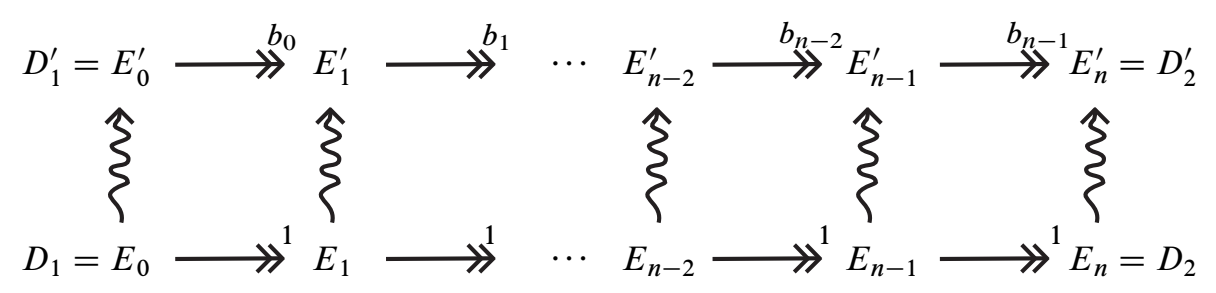

Proposition 3(b) gives us that $b_{i} \leq c\left(E_{i}^{\prime}\right)-c\left(E_{i}\right)$, while Proposition 3(a) tells us $c\left(E_{i}^{\prime}\right)-c\left(E_{i}\right) \leq 2^{i}\left(c\left(E_{0}^{\prime}\right)-c\left(E_{0}\right)\right)$. The sequence of $\Omega_{2}$ moves from $E_{0}^{\prime}$ to $E_{n}^{\prime}$ has length $b$, where $b=\sum_{i=0}^{n-1} b_{i}$. Hence,

$$
b \leq\left(2^{n}-1\right)\left(c\left(E_{0}^{\prime}\right)-c\left(E_{0}\right)\right) .
$$

Take $D_{2}^{\prime}=E_{n}^{\prime}$ to complete the proof.

Theorem 5 below makes use of Lemma 2, Proposition 3 and Corollary 4 to begin building an ordered sequence from an unordered sequence.

Theorem 5 Let $D_{2}$ be a link diagram obtained from $D_{1}$ via a sequence of $\Omega_{2}$ and $\Omega_{3}$ moves of length $M$. Then there exists a diagram $D_{3}$ such that $D_{1} \rightarrow^{c} D_{3}$ with $D_{3}$ is obtained from $D_{2}$ by adding a sequence no more than $M$ tails and lollipops. Further, $c \leq \exp ^{(M)}(6 M)$.

Proof Consider a sequence $\mathcal{A}$ of $\Omega_{2}$ and $\Omega_{3}$ moves of length $M$ from $D_{1}$ to $D_{2}$, of which $N$ are $\Omega_{3}$ :

$$
\mathcal{A}: D_{1}=A_{0} \rightarrow A_{1} \rightarrow \cdots \rightarrow A_{M}=D_{2}
$$

Using Lemma 2 , cap every $\Omega_{3}$ move to build a new sequence $\mathcal{E}_{1}$ :

$$
\mathcal{E}_{1}: D_{1}=E_{0} \rightarrow E_{1} \rightarrow \cdots \rightarrow E_{M+2 N}=D_{2},
$$

where $\mathcal{E}_{1}$ contains no $\Omega_{3}$ moves. This is depicted in Figure 5 .

If $E_{i} \stackrel{\Omega_{2}^{\downarrow}}{\longrightarrow} E_{i+1}$, we relabel this as $E_{i}$ « $E_{i+1}$, because a $\Omega_{2}^{\uparrow}$ move may be performed by adding a tail. Define a local minimum of $\mathcal{E}_{1}$ to be a diagram $E_{i}$ such that

$$
E_{i-1} \text { \& } E_{i} \stackrel{\Omega_{2}^{\uparrow}}{\longrightarrow} E_{i+1} \quad \text { or } \quad E_{i-1} \leftarrow E_{i} \stackrel{\Omega_{2}^{\uparrow}}{\longrightarrow} E_{i+1} .
$$

Let $E_{J} \in\left\{E_{1}, \ldots, E_{M+2 N-1}\right\}$ be the local minimum appearing in $\mathcal{E}_{1}$ with greatest index. Let $r_{1}$ be the number of consecutive $\Omega_{2}^{\uparrow}$ moves in $\mathcal{E}_{1}$ to the right of $E_{J}$. Let $\ell_{1}$ be the number of consecutive $\Omega_{2}^{\uparrow}$ moves in $\mathcal{E}_{1}$ to the left of $E_{J-1}$. 


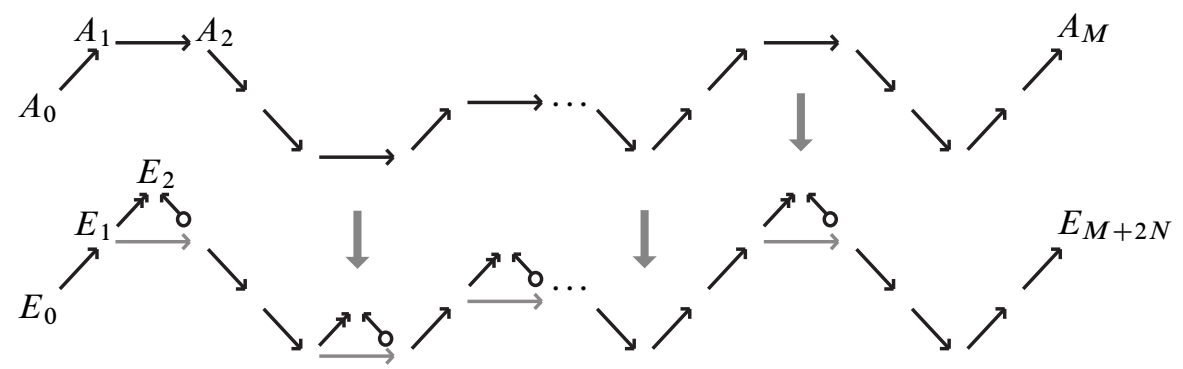

Figure 5: Constructing $\mathcal{E}_{1}$ from $\mathcal{A}$

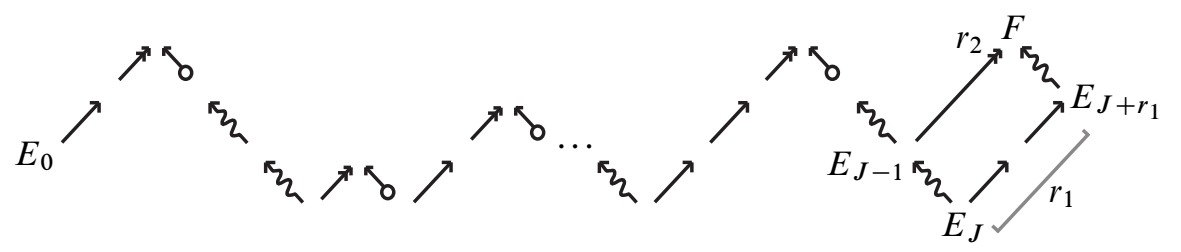

Figure 6: Constructing $F$ (in this case, $E_{J+r_{1}}=E_{M+2 N}$ )

Apply Corollary 4 to the triple $\left(E_{J-1}, E_{J}, E_{J+r_{1}}\right)$ to build a diagram $F$, where $E_{J-1} \rightarrow_{2}^{\prime} F$ and where $E_{J+r_{1}} \bigcirc \rightarrow F$ if $E_{J} \bigcirc \rightarrow E_{J-1}$ or $E_{J+r_{1}} \rightsquigarrow F$ if $E_{J} \rightsquigarrow$ $E_{J-1}$. Corollary 4 tells us $r_{2}^{\prime} \leq 4 \cdot 2^{r_{1}}$, in the worst case that $E_{J} \bigcirc \rightarrow E_{J-1}$. Figure 6 depicts the construction of $F$. Define $\mathcal{E}_{2}$ to be the following sequence:

$$
\mathcal{E}_{2}: D_{1}=E_{0} \rightarrow E_{1} \rightarrow \cdots \rightarrow E_{J-1} \rightarrow \cdots \rightarrow F \rightarrow E_{J+r_{1}} \rightarrow \cdots \rightarrow E_{M+2 N}
$$

Then $\mathcal{E}_{2}$ is a sequence of diagrams with $r_{2}$ consecutive $\Omega_{2}^{\uparrow}$ moves to the right of its last local minimum, where we have the following bound on $r_{2}$ :

$$
r_{2} \leq 4 \cdot 2^{r_{1}}+\ell_{1} \leq 2^{r_{1}+2+\ell_{1}} .
$$

Let $\mathcal{E}_{k}$ be the sequence obtained by $k-1$ applications of Corollary 4, with $r_{k}$ the number of $\Omega_{2}^{\uparrow}$ moves to the right of the last local minimum of $\mathcal{E}_{k}$. Let $\ell_{k}$ be the number of consecutive $\Omega_{2}^{\uparrow}$ moves preceding the diagram to the immediate left of the last local minimum of $\mathcal{E}_{k}$. Given the $\mathcal{E}_{k}$ and $r_{k}$, we may apply Corollary 4 as above to produce a sequence $\mathcal{E}_{k+1}$ and corresponding $r_{k+1}$ with $r_{k+1} \leq 2^{r_{k}+2+\ell_{k}}$, and hence

$$
r_{k+1} \leq \exp ^{(k)}\left(r_{1}+2 k+\sum_{i=1}^{k} \ell_{i}\right) .
$$

Iterate the constructions of the $\left(\mathcal{E}_{k}, r_{k}\right)$ until we produce a sequence $\mathcal{E}_{K}$ with no local minima and with $r_{K}$ consecutive $\Omega_{2}^{\uparrow}$ moves following $E_{0}$. The number of times we 


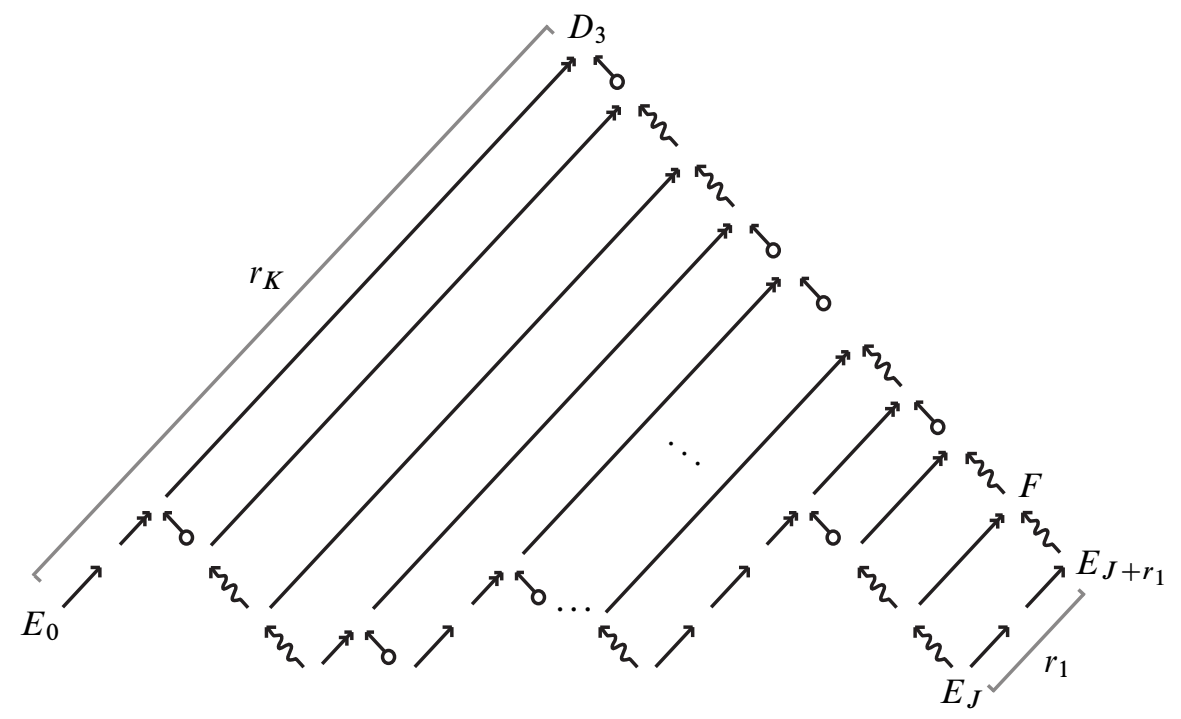

Figure 7: Repeatedly applying Corollary 4 to build $D_{3}$

apply Corollary 4 to construct $\mathcal{E}_{K}$ from $\mathcal{E}_{1}$ is exactly the number of tails and lollipops in $\mathcal{E}_{1}$, which is less than or equal to $M$. So $K \leq M+1$, and via (1) above,

$$
r_{K} \leq \exp ^{(M)}(6 M)
$$

where we've used that $r_{1} \leq M$ and $\sum_{i=1}^{K-1} \ell_{i} \leq M+2 N \leq 3 M$.

There are $r_{K}$ moves of type $\Omega_{2}^{\uparrow}$ following $D_{1}=E_{0}$ in $\mathcal{E}_{K}$, so let $D_{3}$ be the diagram obtained by performing these moves on $D_{1}$. Because $D_{3}$ is obtained from $E_{M+2 N}=$ $D_{2}$ by at most $M$ tails and lollipops, Theorem 5 holds.

The following theorem allows us to construct an ordered sequence of $\Omega_{2}$ and $\Omega_{3}$ moves from the tails and lollipops arising in Theorem 5.

Theorem 6 Suppose $D_{2}$ is obtained from $D_{1}$ by a sequence $\mathcal{T}$ of tails and lollipops of length $M$ :

$$
\mathcal{T}: D_{1}=T_{0} \stackrel{a_{1}}{\longrightarrow} T_{1} \stackrel{a_{2}}{\longrightarrow} \cdots \stackrel{a_{M}}{\longrightarrow} T_{M}=D_{2}
$$

where either $T_{i} \rightsquigarrow T_{i+1}$ or $T_{i} \bigcirc T_{i+1}$. Then there exists a diagram $D_{3}$ obtained from $D_{2}$ by a sequence of $\Omega_{2}^{\uparrow}$ moves of length no more than $\frac{M}{2}\left(c\left(D_{2}\right)-c\left(D_{1}\right)\right)+2 M$, followed by a sequence of $\Omega_{3}$ moves of length no more than $M$. Additionally $D_{1}$ is obtained from $D_{3}$ by a sequence of $\Omega_{2}^{\downarrow}$ moves of length at most $\frac{M+1}{2}\left(c\left(D_{2}\right)-\right.$ $\left.c\left(D_{1}\right)\right)+2 M$. 
Proof Consider a crossing $\chi$ of the diagram $D_{2}$ about which the circle part of any lollipop in $\mathcal{T}$ is centered. There may be multiple lollipops (suppose there are $k$ ) centered at $\chi$, so consider a point $p_{k}$ on the outermost one. Let $q$ be a point in a small enough neighborhood of $\chi$ such that a straight line segment from $q$ to $\chi$ does not intersect $D_{2}$ except at $\chi$.

Consider a path $c:[0,1] \rightarrow \mathbb{R}^{2}$ such that $c(0)=p_{k}$ and $c(1)=q$. Choose $c$ in such a way that its image $C$ intersects each concentric lollipop at only one point. The point of intersection of $C$ and the $i$ th concentric lollipop is denoted $p_{i}$. Let $\delta_{k}=0$ and let $\delta_{k-1}<\delta_{k-2}<\cdots<\delta_{1} \in(0,1)$ such that $c\left(\delta_{i}\right)=p_{i}$.
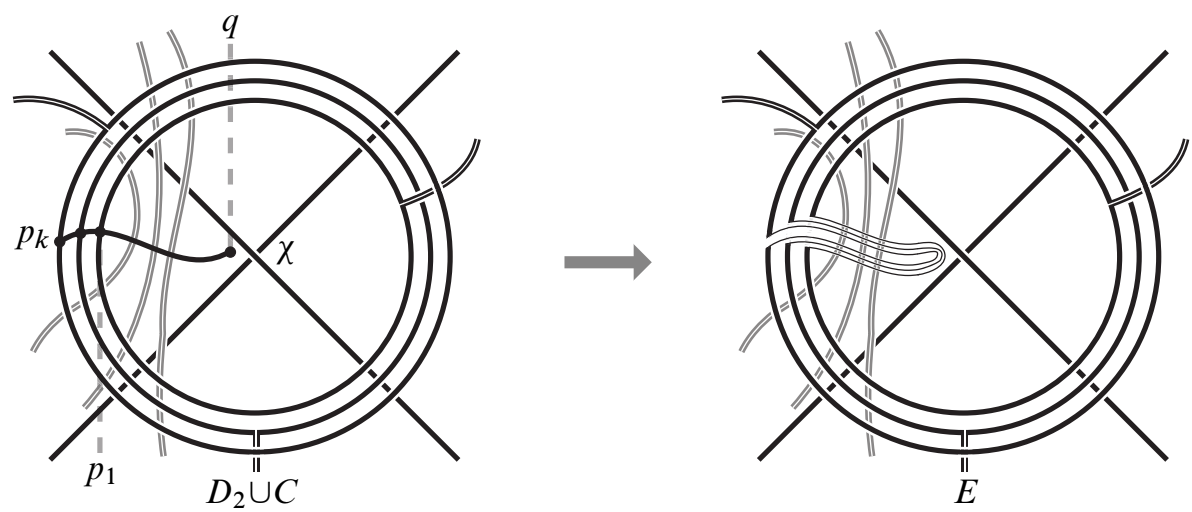

Figure 8: Adding concentric tails at the crossing $\chi$

As in the proof of Proposition 3, we also choose $c$ so that $C \cap D_{2}$ consists of no more than $2\left\lfloor\frac{c\left(D_{2}\right)-c\left(D_{1}\right)}{4}\right\rfloor$ points, excluding the points $p_{1}$ through $p_{k}$.

Add a tail along the path $\left.c\right|_{\left[\delta_{1}, 1\right]}$ to construct a diagram $E_{1}$ from $D_{2}$, where $c\left(E_{1}\right)-$ $c\left(D_{2}\right) \leq c\left(D_{2}\right)-c\left(D_{1}\right)$. Perturb this tail slightly, so that it is closer to the crossing $\chi$, and now add a second tail disjoint from the first tail along the path $\left.c\right|_{\left[\delta_{2}, 1\right]}$. This second tail introduces no more than $c\left(D_{2}\right)-c\left(D_{1}\right)$ crossings.

Repeating this process of perturbing and adding tails along $\left.c\right|_{\left[\delta_{i}, 1\right]}$ for all $i \in\{1, \ldots, k\}$, we produce a diagram $E_{k}$ where $c\left(E_{k}\right)-c\left(D_{2}\right) \leq k\left(c\left(D_{2}\right)-c\left(D_{1}\right)\right)$. We then build the diagram $E$ by adding nested tails in the same way for every crossing of $D_{2}$ that is the center of some lollipop, so that $c(E)-c\left(D_{2}\right) \leq M\left(c\left(D_{2}\right)-c\left(D_{1}\right)\right)$. The construction of $E$ is depicted in Figure 8. The diagram $E$ may be obtained from $D_{2}$ by a sequence of $\Omega_{2}^{\uparrow}$ moves of length at most $\frac{M}{2}\left(c\left(D_{2}\right)-c\left(D_{1}\right)\right)$.

Now construct the diagram $E^{\prime}$ from $E$ by performing the following at each crossing: if there are $k$ concentric circles centered at a crossing $\chi$, perform $2 k$ type $\Omega_{2}^{\uparrow}$ moves, 


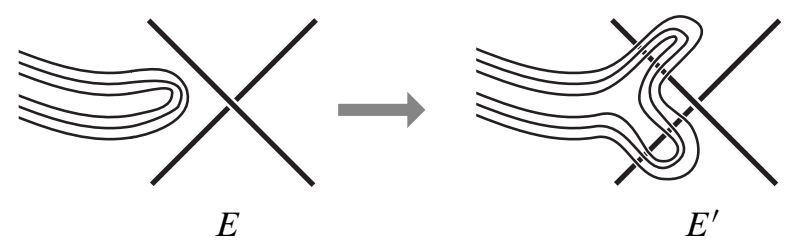

Figure 9: Perform $2 k$ type $\Omega_{2}^{\uparrow}$ moves, so that each tail 'forks' over the crossing

forking the previously constructed tails over the edges of the crossing $\chi$, as Figure 9 illustrates.

The diagram $E^{\prime}$ may be reached from $D_{2}$ via a sequence of $\Omega_{2}^{\uparrow}$ moves with length at most $\frac{M}{2}\left(c\left(D_{2}\right)-c\left(D_{1}\right)\right)+2 M$. Finally, construct the diagram $D_{3}$ by performing at most $M$ moves of type $\Omega_{3}$, as in Figure 10 .

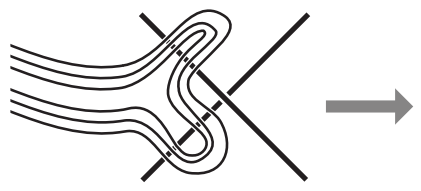

$E^{\prime}$

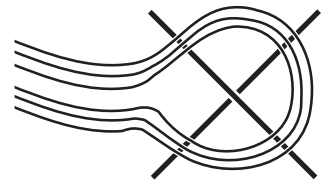

$D_{3}$

Figure 10: Performing $\Omega_{3}$ moves to pass from $E^{\prime}$ to $D_{3}$

We may now pass from $D_{3}$ to $D_{1}$ by performing $\Omega_{2}^{\downarrow}$ moves as follows. Each tail and lollipop of $\mathcal{T}$ in $D_{2}$ is still present in $D_{3}$, with the circle parts of each lollipop modified. We remove them one at a time starting with the last tail or lollipop $a_{M}$ in the sequence. If $a_{M}$ is a lollipop, it now has the form depicted by Figure 11 in $D_{3}$, and may be removed by $\Omega_{2}^{\downarrow}$ moves. If $a_{M}$ is a tail, it may likewise be removed by $\Omega_{2}^{\downarrow}$ moves. We continue to remove tails and lollipops in the reverse order they are added in $\mathcal{T}$ until we obtain $D_{1}$.

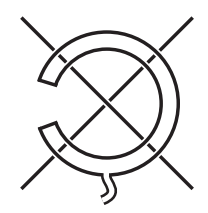

Figure 11

Because $c\left(D_{3}\right)=c\left(E^{\prime}\right)$, we know $c\left(D_{3}\right)-c\left(D_{1}\right)$ is exactly $c\left(E^{\prime}\right)-c\left(D_{2}\right)+c\left(D_{2}\right)-$ $c\left(D_{1}\right)$, which is at most $M\left(c\left(D_{2}\right)-c\left(D_{1}\right)\right)+4 M+c\left(D_{2}\right)-c\left(D_{1}\right)$. Halving this gives us a bound on the number of $\Omega_{2}^{\downarrow}$ moves from $D_{3}$ to $D_{1}$. 
We consolidate previous results into Theorem 7, a special case of Theorem 1 .

Theorem 7 Let $D_{2}$ be a link diagram obtained from $D_{1}$ by a sequence of $\Omega_{2}$ and $\Omega_{3}$ moves of length $M$. Then there is a sequence of at most $\exp ^{(2 M)}(6 M)$ Reidemeister moves from $D_{1}$ to $D_{2}$ ordered in the following way: first $\Omega_{2}^{\uparrow}$ moves, then $\Omega_{3}$ moves and finally $\Omega_{2}^{\downarrow}$ moves.

Proof Given $D_{1}$ and $D_{2}$, construct a diagram $D_{3}$ using Theorem 5, where $D_{3}$ is obtained from $D_{1}$ by no more than $\exp ^{(M)}(6 M)$ type $\Omega_{2}^{\uparrow}$ moves, and where $D_{3}$ is obtained from $D_{2}$ by no more than $M$ tails and lollipops. Note that $c\left(D_{3}\right)-c\left(D_{2}\right) \leq$ $2 \cdot \exp ^{(M)}(6 M)+2 M$.

From $D_{2}$ and $D_{3}$, apply Theorem 6 to construct a diagram $D_{4}$ with the following properties: there is a sequence of $\Omega_{2}^{\uparrow}$ moves whose length is no more than $M$. $\exp ^{(M)}(6 M)+M^{2}+2 M$, followed by a sequence of $\Omega_{3}$ moves of length no more than $M$ from $D_{3}$ to $D_{4}$. There is also a sequence of $\Omega_{2}^{\downarrow}$ moves whose length is at most $(M+1) \cdot \exp ^{(M)}(6 M)+M^{2}+3 M$ from $D_{4}$ to $D_{2}$.

Following the sequences of moves constructed from $D_{1}$ to $D_{3}$, then to $D_{4}$ and finally to $D_{2}$, we have a sequence of no more than $(2 M+2) \cdot \exp ^{(M)}(6 M)+M(2 M+6)$ Reidemeister moves ordered as desired. For $M \geq 1$, $\exp ^{(2 M)}(6 M) \geq(2 M+2)$. $\exp ^{(M)}(6 M)+M(2 M+6)$.

Before considering the more general case of an arbitrary sequence of $M$ Reidemeister moves, we need two lemmas relating to $\Omega_{1}$ moves. These lemmas allow us to take a sequence of Reidemeister moves and build a new sequence in which the $\Omega_{1}$ moves occur only at the beginning and end.

Lemma 8 Let $A, B$ and $C$ be link diagrams such that

$$
A \stackrel{\Omega}{\rightarrow} B \stackrel{\Omega_{1}^{\uparrow}}{\longrightarrow} C
$$

where $\Omega$ is an arbitrary $\Omega_{2}$ or $\Omega_{3}$ move. Then there exists a diagram $B^{\prime}$ which may be obtained from $A$ by a single $\Omega_{1}^{\uparrow}$ move, and where $C$ is obtained from $B^{\prime}$ by no more than six $\Omega_{2}$ or $\Omega_{3}$ moves. Additionally, if instead $\Omega=\Omega_{1}^{\downarrow}$, there is a diagram $B^{\prime}$ such that

$$
A \stackrel{\Omega_{1}^{\uparrow}}{\longrightarrow} B^{\prime} \stackrel{\Omega_{1}^{\downarrow}}{\longrightarrow} C .
$$

Lemma 9 Let $A, B$ and $C$ be link diagrams such that $A \stackrel{\Omega_{1}^{\downarrow}}{\longrightarrow} B \stackrel{\Omega}{\rightarrow} C$, where $\Omega$ is an $\Omega_{2}$ or $\Omega_{3}$ move. Then there exists a diagram $B^{\prime}$ such that $B^{\prime}$ is obtained from $A$ by no more than six $\Omega_{2}$ or $\Omega_{3}$ moves and where $C$ may be obtained from $B^{\prime}$ by a single $\Omega_{1}^{\downarrow}$ move. 
The proofs of Lemma 8 and Lemma 9 are left to be verified by the reader, and Corollary 10 is a rapid consequence of these lemmas:

Corollary 10 Let $D_{2}$ be obtained from $D_{1}$ by an arbitrary sequence of $M$ Reidemeister moves, $\alpha$ of which are $\Omega_{1}^{\uparrow}$ and $\beta$ of which are $\Omega_{1}^{\downarrow}$. Then there exist diagrams $D_{1}^{\prime}$ and $D_{2}^{\prime}$ such that $D_{1}^{\prime}$ is obtained from $D_{1}$ by $\alpha$ type $\Omega_{1}^{\uparrow}$ moves and $D_{2}$ is obtained from $D_{2}^{\prime}$ by $\beta$ type $\Omega_{1}^{\downarrow}$ moves. Additionally, $D_{2}^{\prime}$ is obtained from $D_{1}^{\prime}$ by no more than $6^{M} M$ Reidemeister moves of type $\Omega_{2}$ and $\Omega_{3}$.

We conclude by proving Theorem 1 .

Proof of Theorem 1 Begin with an arbitrary sequence of $M$ Reidemeister moves from diagram $D_{1}$ to diagram $D_{2}, \alpha$ of which are $\Omega_{1}^{\uparrow}$ and $\beta$ of which are $\Omega_{1}^{\downarrow}$. Construct $D_{1}^{\prime}$ and $D_{2}^{\prime}$ as in Corollary 10. Apply Theorem 7 to the sequence of $\Omega_{2}$ and $\Omega_{3}$ moves from $D_{1}^{\prime}$ to $D_{2}^{\prime}$ to obtain a sorted sequence of Reidemeister moves from $D_{1}$ to $D_{2}$ of length at most

$$
\exp ^{\left(2 \cdot 6^{M} M\right)}\left(6 \cdot 6^{M} M\right)+\alpha+\beta \leq \exp ^{\left(6^{M+1} M\right)}\left(6^{M+1} M\right) .
$$

Acknowledgements I am extraordinarily grateful to Alexander Coward for many insightful discussions and for his guidance in writing this paper. This research was partially supported by NSF VIGRE grant DMS0636297.

\section{References}

[1] A Coward, Ordering the Reidemeister moves of a classical knot, Algebr. Geom. Topol. 6 (2006) 659-671 MR2240911

[2] A Coward, M Lackenby, Unknotting genus one knots, Comment. Math. Helv. 86 (2011) 383-399 MR2775133

[3] J Hass, J C Lagarias, The number of Reidemeister moves needed for unknotting, J. Amer. Math. Soc. 14 (2001) 399-428 MR1815217

[4] B Trace, On the Reidemeister moves of a classical knot, Proc. Amer. Math. Soc. 89 (1983) 722-724 MR719004

\section{UCLA Mathematics Department}

Box 951555, Los Angeles, CA 90095-1555, USA

jugold@ucla.edu

Received: 17 April 2012 